\title{
The testis in patients with COVID-19: virus reservoir or immunization resource?
}

\author{
Federica Barbagallo ${ }^{1}$, Aldo E. Calogero ${ }^{1}$, Rossella Cannarella ${ }^{1}$, Rosita A. Condorelli ${ }^{1}$, Laura M. Mongioì ${ }^{1}$, \\ Antonio Aversa ${ }^{2}$, Sandro La Vignera ${ }^{1}$ \\ ${ }^{1}$ Department of Clinical and Experimental Medicine, University of Catania, Catania, Italy; ${ }^{2}$ Department of Experimental and Clinical Medicine, \\ University Magna Graecia of Catanzaro, Catanzaro, Italy \\ Correspondence to: Dr. Rosita A. Condorelli. Department of Clinical and Experimental Medicine, University of Catania, via S. Sofia 78, 95123 Catania, \\ Italy. Email: rosita.condorelli@unict.it.
}

Submitted Apr 28, 2020. Accepted for publication Jul 06, 2020.

doi: $10.21037 /$ tau-20-900

View this article at: http://dx.doi.org/10.21037/tau-20-900

In December 2019, a novel coronavirus designated SARS-CoV-2 was identified in patients with pneumonia in Wuhan, Hubei Province of China (1). The World Health Organization (WHO) assigned the official name "COVID-19" to the new coronavirus disease and on the 11th of March 2020, COVID-19 was declared a global pandemic. The major morbidity and mortality from COVID-19 is due to acute viral pneumonitis that evolves to acute respiratory distress syndrome (ARDS) (2).

SARS-CoV- 2 shares about $76 \%$ of amino acid sequence with severe acute respiratory syndrome coronavirus (SARS-CoV) and it uses the same receptor of SARS-CoV, angiotensin-converting enzyme 2 (ACE2) for entry into target host cells (3). ACE2 is a component of the reninangiotensin system (RAS). SARS-CoV-2 infection decreases ACE2 activity, exacerbating pathophysiological mechanisms, such as angiotensin II/ACE2 regulation imbalance (4). ACE2 is highly expressed in type II alveolar epithelial cells (AT2) in human lungs (5) suggesting that COVID-19 targets ACE2-positive AT2 cells to induce pneumonia. However, it was shown that ACE2 is also expressed in the liver, specifically in cholangiocytes (6) and in the digestive system, specifically in the upper esophagus and stratified epithelial cells and in the absorptive enterocytes in the ileum and colon (7). In addition, cardiovascular and urinary systems have been reported as potential organ targets of SARS-CoV-2 infection (8). It was described that respiratory, cardiovascular, digestive and urinary systems are affected by SARS-CoV-2 infection $(7,8)$.

Fan and colleagues clearly showed that ACE2 is also expressed in adult human testes (9). Based on scRNAseq profiling of human testes, Wang and Xu reported that ACE2 is highly expressed in spermatogonia and Leydig and Sertoli cells. Interestingly, ACE2 expression in Leydig and Sertoli cells is about 3-fold higher than that present in AT2 cells that express this enzyme (4.25\% versus $1.40 \%$ ) (1). In addition, a recent study reported that SARS-CoV-2 uses the transmembrane serine protease 2 (TMPRSS2) for viral spike (S) protein priming (3). It was found that TMPRSS2 is highly expressed in spermatogonia and spermatids (1). Gene ontology (GO) enrichment analysis further indicated that GO categories associated with viral reproduction and transmission were positively enriched in ACE2-positive spermatogonia (e.g., NUP133, POLR2A, JUN, RANBP2, RPL12, EIF3L, RPL3, RPL4, RPS19, RPS2, NUP85). In contrast, genes that are involved in spermatogenesis are less expressed in ACE2-positive cells. Thus, the authors hypothesized that SARS-CoV-2 may directly target ACE2positive spermatogonia and disrupt spermatogenesis. In addition, genes involved in cell junctions and immunity were higher expressed in ACE2-positive Leydig and Sertoli cells compared to ACE2 negative cells. This finding suggests that SARS-CoV-2 may replicate and transfer through cell-cell junctions (1).

However, there is no clinical evidence about the effects of SARS-CoV-2 on male gonadal function. Ma and colleagues evaluated sexual hormones in 81 men in reproductive age with SARS-CoV-2 infection (10). They found that serum luteinizing hormone (LH) levels were significantly higher and the ratio testosterone $(\mathrm{T}) / \mathrm{LH}$ lower in patients 
with COVID-19 then in healthy men. These results are compatible with an early stage primary hypogonadism, in which impaired $\mathrm{T}$ production by Leydig cells may stimulate the release of $\mathrm{LH}$ that maintains $\mathrm{T}$ level temporarily in the normal range (10). However, the small samples, the different disease severity of patient enrolled, the different therapy used, and the short length of time of these observations may have influenced the results. Therefore, further studies are needed to confirm these findings. Unfortunately, to our knowledge, no data on sperm parameters have been reported in patients with COVID-19.

COVID-19 is a concern for couples seeking pregnancy. After binding with ACE2-positive cells, SARS-CoV-2 penetrate within the testes that could become a viral reservoir (11). Numerous viruses, including HIV, mumps virus, Hepatitis B and C viruses, Epstein-Barr virus, Papilloma virus, and SARS-CoV may damage the testis (12). Some of them, like HIV, HBV, HSV, and adenovirus, are also found in the semen (13). Rothe and collaborators first reported that the SARS-CoV2 infection can be transmitted during the incubation period when the patient did not have any symptoms. This case-report raises the question about possible new routes of transmission (14). However, very recently, Song and colleagues reported that SARS-CoV-2 is not present in spermatozoa of 12 recovered COVID-19 patients. Also, the virus was not found in the testis of one patient who died during the acute phase. However, the sample size is very small and more patients are needed to better evaluate the presence of SARS-CoV-2 in the seminal fluid or the testis of infected patients (15). The American Society for Reproductive Medicine (ASRM) and the European Society of Human Reproduction and Embryology (ESHRE) have advised that prospective parents, couples who wish to undergo assisted reproductive techniques (ART), gamete donors, and gestational carriers infected with SARS-CoV-2 should avoid becoming pregnant or participate in any fertility programs. The situation is highly fluid and the guidance for ART patients are revisited periodically as the pandemic evolves (16).

Since SARSCoV2 and SARS-CoV have a similar structure, it has been hypothesized that they may share analogous pathogenesis and pathological manifestations. Previous studies have shown that orchitis is a complication of SARS (12). SARS-infected patients have testicular damage and spermatogenesis abnormalities. Testes of SARS patients have leukocyte infiltration, basement thickening, peritubular fibrosis, vascular congestion and loss of gem cells. Immunohistochemical analysis showed significant higher levels of $\mathrm{CD}^{+} \mathrm{T}$ lymphocytes and $\mathrm{CD} 68^{+}$ macrophages in the testis of SARS patients compared with the controls. The authors hypothesized that these cells could impair Leydig cell function (consequently testosterone production), blood-testis barrier (BTB), and damage seminiferous epithelium directly. In addition, the inflammatory process may trigger an autoimmune response and autoantibody production within the tubules. Although the testis is an immunologically privileged organ because of the present of the BTB, numerous immune-regulatory molecules can be secreted by testicular cells (17). Models of experimental autoimmune orchitis showed deposits of IgG in germinal epithelium, basement membrane, interstitium, vascular endothelium, and degenerated germ cells (18). Interestingly, an extensive IgG precipitation in the seminiferous epithelium, including in some degenerated germ cells and Sertoli cells, has been reported in patients with SARS (12). Figure 1 shows the putative mechanisms of action of SARS-CoV-2 within the testis and its pathophysiological consequences.

Humoral immune response, especially the production of neutralizing antibody, has a crucial role for the limitation of the infection in later phases and to prevent re-infection (19). In $89 \%$ of patients who recovered form SARS, IgG-specific and neutralizing antibodies were found two years after infection (20). COVID-19 stimulates the production of IgG against the $\mathrm{N}$ protein that can be measured in the serum as early as day 4 after the onset of disease and most of the patients seroconvert within the second week of infection (19). The analysis of ACE2 in lung cells of patients with COVID-19 suggests that this enzyme is not only a receptor, but is also involved in post-infection regulation, including innate immune response, adaptive immune response, cytokine secretion, and viral genome replication (21). Since the high expression of ACE2 receptors in the testis $(1,9)$, this organ could play an important role in the adaptive immune response to COVID-19.

In conclusion, evidence suggests that the testis may be vulnerable to SARS-CoV2 infection. Thus, the reproductive function should be followed and evaluated in COVID-19 male patients, especially in young men. At the same time, the susceptibility of males to severe disease has been constantly reported in these months and men show worse clinical outcomes than women. Although the etiology is probably multifactorial, the effects of sex hormones on the expression of ACE2 could help in explaining this different susceptibility and lethality between sexes (22). Finally, we speculate that the male gonad may have a potentially 


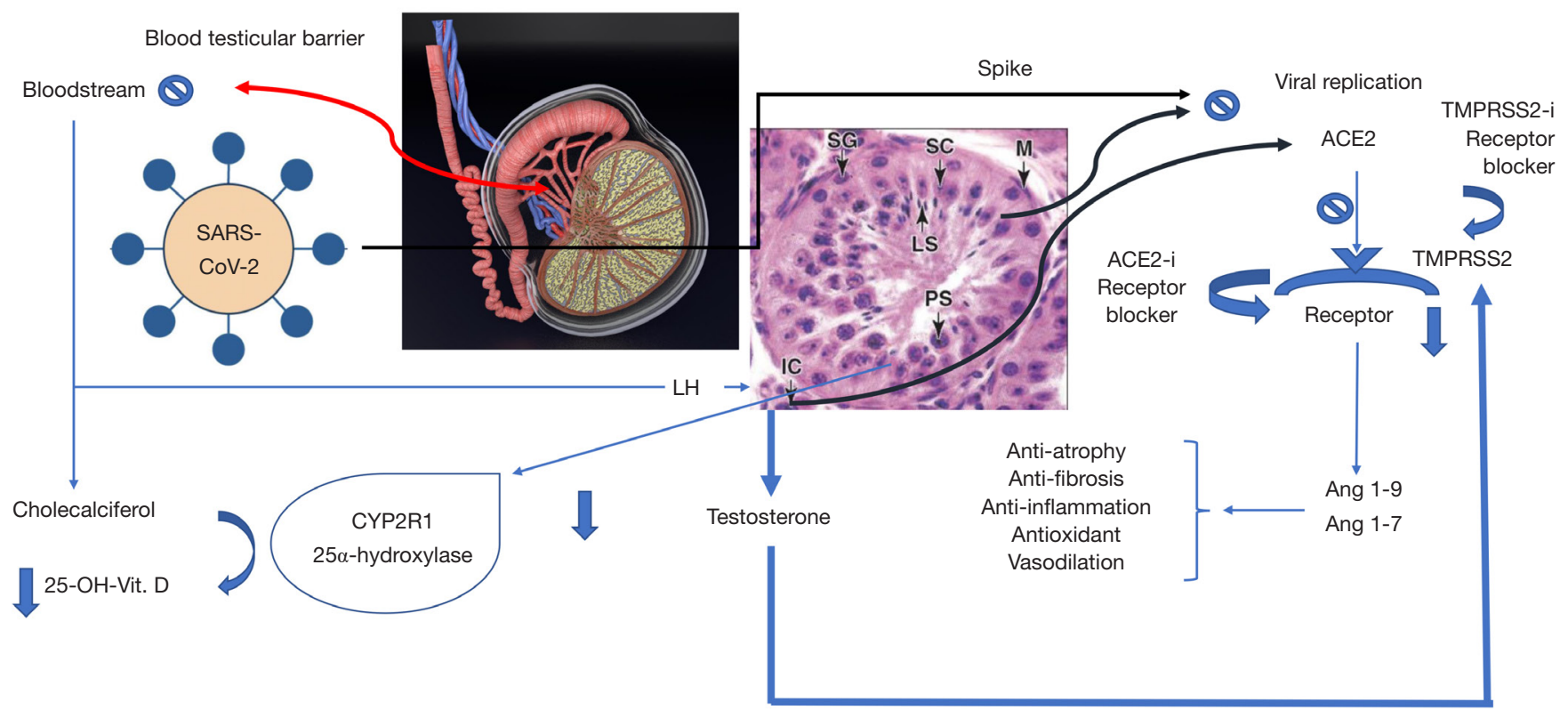

Figure 1 Putative mechanisms of action of SARS-CoV-2 within the testis. Viral spike protein may penetrate the blood-testicular-barrier and, after putative replication, may interact with ACE2 receptors expressed in Leydig and germ cells. The binding of SARS-CoV-2 spike protein to ACE2 down-regulates downstream receptor, thus decreasing its beneficial effects on atrophy/fibrosis/inflammation exerted through Ang 1-9 and Ang 1-7 activation. Also, the possible presence of reduced secretion of LH that frequently such as in patients with hypothalamic-pituitary dysfunction, metabolic hypogonadism, and late-onset hypogonadism, may further contribute in the establishment of low testosterone levels and vitamin D 25-hydroxylation. This is an important protective mechanism leading to TMPRSS2 activation, which has a pivotal role in regulating virus entry into the testis. Finally, when compensatory mechanisms fail, orchitis determined by IgG precipitation in the seminiferous epithelium of SARS testes may represent a complication of SARS [Xu J, Qi L, Chi X, Yang J, Wei X, Gong E, Peh S, Gu J. Orchitis: a complication of severe acute respiratory syndrome (SARS) 2006;74:410-6] and suggests that the reproductive functions should be followed and evaluated in recovered male COVID-19 patients.

important role in the onset of adaptive immune response to COVID-19. It would be interesting to know the disease course for COVID-19 patients with a normal gonadal function or with previous testicular pathologies. Few data are still available about the link between COVID-19 and male reproductive system but many questions are already open and need to be answered.

\section{Acknowledgments}

Funding: None.

\section{Footnote}

Provenance and Peer Review: This article was a free submission to the journal. The article did not undergo external peer review.
Conflicts of Interest: All authors have completed the ICMJE uniform disclosure form (available at http://dx.doi. org/10.21037/tau-20-900). The authors have no conflicts of interest to declare.

Ethical Statement: The authors are accountable for all aspects of the work in ensuring that questions related to the accuracy or integrity of any part of the work are appropriately investigated and resolved.

Open Access Statement: This is an Open Access article distributed in accordance with the Creative Commons Attribution-NonCommercial-NoDerivs 4.0 International License (CC BY-NC-ND 4.0), which permits the noncommercial replication and distribution of the article with the strict proviso that no changes or edits are made and the original work is properly cited (including links to both the 
formal publication through the relevant DOI and the license). See: https://creativecommons.org/licenses/by-nc-nd/4.0/.

\section{References}

1. Wang Z, Xu X. scRNA-seq Profiling of Human Testes Reveals the Presence of the ACE2 Receptor, A Target for SARS-CoV-2 Infection in Spermatogonia, Leydig and Sertoli Cells. Cells 2020;9:920.

2. Chen N, Zhou M, Dong X, et al. Epidemiological and clinical characteristics of 99 cases of 2019 novel coronavirus pneumonia in Wuhan, China: a descriptive study. Lancet 2020;395:507-13.

3. Hoffmann M, Kleine-Weber H, Schroeder S, et al. SARSCoV-2 Cell Entry Depends on ACE2 and TMPRSS2 and Is Blocked by a Clinically Proven Protease Inhibitor. Cell 2020;181:271-80.e8.

4. Cheng H, Wang Y, Wang GQ. Organ-protective effect of angiotensin-converting enzyme 2 and its effect on the prognosis of COVID-19. J Med Virol 2020;92:726-30.

5. Zhao Y, Zhao Z, Wang Y, et al. Single-cell RNA expression profiling of ACE2, the putative receptor of Wuhan 2019nCov. Available online: https://www.biorxiv.org/content/1 $0.1101 / 2020.01 .26 .919985 \mathrm{v} 1$

6. Chai X, Hu L, Zhang Y, et al. Specific Ace2 Expression in cholangiocytes may cause liver damage after 2019-Ncov infection. Available online: https://www.biorxiv.org/conten $\mathrm{t} / 10.1101 / 2020.02 .03 .931766 \mathrm{v} 1$

7. Zhang H, Kang Z, Gong H, et al. The digestive system is a potential route of 2019-nCov infection: a bioinformatics analysis based on single-cell transcriptomes. Available online: https://www.biorxiv.org/content/10.1101/2020.01. 30.927806v1

8. Zou X, Chen K, Zou J, et al. Single-cell RNA-seq data analysis on the receptor ACE2 expression reveals the potential risk of different human organs vulnerable to 2019-nCoV infection. Front Med 2020;14:185-92.

9. Fan C, Li K, Ding Y, et al. ACE2 expression in kidney and testis may cause kidney and testis damage after 2019-nCoV infection. Available online: https://www.medrxiv.org/conte $\mathrm{nt} / 10.1101 / 2020.02 .12 .20022418 \mathrm{v} 1$

10. Ma L, Xie W, Li D, et al. Effect of SARS-CoV-2 infection upon male gonadal function: A single center-based study. Available online: https://www.medrxiv.org/content/10.110 $1 / 2020.03 .21 .20037267 \mathrm{v} 2$

11. Cardona Maya WD, Du Plessis SS, Velilla PA. SARS$\mathrm{CoV}-2$ and the testis: similarity to other viruses and routes of infection. Reprod Biomed Online 2020;40:763-4.

12. Xu J, Qi L, Chi X, et al. Orchitis: a complication of severe acute respiratory syndrome (SARS). Biol Reprod 2006;74:410-6.

13. Dejucq N, Jégou B. Viruses in the mammalian male genital tract and their effects on the reproductive system. Microbiol Mol Biol Rev 2001;65:208-31.

14. Rothe C, Schunk M, Sothmann P, et al. Transmission of 2019-nCoV Infection from an Asymptomatic Contact in Germany. N Engl J Med 2020;382:970-1.

15. Song C, Wang Y, Li W, et al. Absence of 2019 novel coronavirus in semen and testes of COVID-19 patients†. Biol Reprod 2020;103:4-6.

16. La Marca A, Niederberger C, Pellicer A, et al. COVID-19: lessons from the Italian reproductive medical experience. Fertil Steril 2020;113:920-2.

17. Qu N, Ogawa Y, Kuramasu M, et al. Immunological microenvironment in the testis. Reprod Med Biol 2019;19:24-31.

18. Itoh $M$, Hiramine $C$, Tokunaga $Y$, et al. A new murine model of autoimmune orchitis induced by immunization with viable syngeneic testicular germ cells alone. II. Immunohistochemical findings of fully-developed inflammatory lesion. Autoimmunity 1991;10:89-97.

19. Rokni M, Ghasemi V, Tavakoli Z. Immune responses and pathogenesis of SARS-CoV-2 during an outbreak in Iran: Comparison with SARS and MERS. Rev Med Virol 2020. doi: 10.1002/rmv.2107.

20. Liu W, Fontanet A, Zhang PH, et al. Two-year prospective study of the humoral immune response of patients with severe acute respiratory syndrome. J Infect Dis 2006;193:792-5.

21. Li G, He X, Zhang L, et al. Assessing ACE2 expression patterns in lung tissues in the pathogenesis of COVID-19. J Autoimmun 2020;112:102463.

22. La Vignera S, Cannarella R, Condorelli RA, et al. SexSpecific SARS-CoV-2 Mortality: Among HormoneModulated ACE2 Expression, Risk of Venous Thromboembolism and Hypovitaminosis D. Int J Mol Sci 2020;21:2948.

Cite this article as: Barbagallo F, Calogero AE, Cannarella R, Condorelli RA, Mongioì LM, Aversa A, La Vignera S. The testis in patients with CoVID-19: virus reservoir or immunization resource? Transl Androl Urol 2020;9(5):1897-1900. doi:10.21037/tau-20-900 